International Journal of Applied Mathematics

Volume 28 No. $6 \quad 2015,789-797$

ISSN: 1311-1728 (printed version); ISSN: 1314-8060 (on-line version)

doi: http://dx.doi.org/10.12732/ijam.v28i6.11

\title{
INFLUENCE OF THE INTERFACIAL STIFFNESS CONSTANTS ON LAMB WAVE PROPAGATION IN A BI-LAYER
}

\author{
M.S.L.R. Mallika ${ }^{1}$, S. Anand Kumar², G. Sudheer ${ }^{3} \S$ \\ ${ }^{1,2}$ Anil Neerukonda Institute of Technology and Sciences \\ Sangivalasa, Visakhapatnam, 531162, INDIA \\ ${ }^{3}$ GVP College of Engineering for Women \\ Madhurawada, Visakhapatnam, 530048, INDIA
}

\begin{abstract}
The interfacial mechanical properties between solid bodies in contact can be effectively monitored using ultrasonic guided waves. The contact interface can be effectively modeled as a spring-type interface that has equivalent stiffness in the normal and tangential directions. The influence of the spring stiffnesses constants on the symmetric and antisymmetric modes of propagation is analyzed in the present work.
\end{abstract}

AMS Subject Classification: 74A50, 74B05, 74H45, 74M15

Key Words: spring model, Lamb waves, interface, adhesive bond

\section{Introduction}

Interfaces play an important role in determining the performance of structural materials on a wide variety of dimension scales ranging from inter-granular boundaries in metals, to inter laminar bonds in composites and adhesively bonded structures Delsanto et al. [4]. Assessing the quality of interfaces is of utmost importance in the design and evaluation of bonded structures and acoustic-based methods provide a non-destructive mean to probe the interface quality Brasier et al. [12]. Modeling of interfacial quality can be tackled us-

$\begin{array}{ll}\text { Received: August 27, } 2015 & \text { (c) } 2015 \text { Academic Publications }\end{array}$

${ }^{\S}$ Correspondence author 
ing different approaches such as introducing dynamic boundary conditions at contact interfaces or introducing a small layer of finite thickness with effective elastic properties. Ideally, a perfect interface is a surface across which both displacement and stress vectors are continuous. These continuity conditions need to be modified in any realistic model of an imperfect interface. A review of the early work on imperfect plane interfaces is available in Martin [7]. The macro mechanics and acoustic signature of imperfect contact surfaces has often been modeled within the frame work of a quasi-static approximation Baik et al. [2] in which the mechanics of the collection of contacts is simulated by that of a homogeneous distribution of springs. The stiffness of the distributed springs can be used to model various imperfect interface conditions with the quality of the interface described by a spring model leading to a discontinuity of the displacement fields and continuity of the stresses across the interface. This is to say that the micro-mechanical behaviour of contacting surfaces is effectively explained on the macroscale by the normal and tangential interfacial stiffness, which are employed to model the stress-displacement system of the contact surfaces as a simple spring-force-displacement system Nam et al. [9] It is argued that the spring model is more reasonable in a physical intrinsic sense to model thin bond layers because the model does not alter the adjoint features of the elastic operator Jun et al. [5]. In Lekesiz et al. [6], analytic expressions for the normal and transverse effective spring stiffnesses of a planar periodic array of collinear cracks at an interface is obtained. In Chuan Her et al. [11],the feasibility of assessing the adhesive bond strength with the ultrasonic technique is carried out. The spring model was used to demonstrate that for a particular adhesive used, the interfacial stiffness measured by the ultrasonic method can be correlated with the adhesive bond strength determined from a tensile test. It is well known that the normal and tangential stiffnesses of the contact interface influence the distinct modes of ultrasonic wave propagation separately Biwa et al. [3]. The effect of each spring stiffness constant on the zero-group velocity Lamb modes in a bi-layer structure has been recently studied in Mezil et al. [8]. The present work is an attempt to study the influence of the stiffness parameters separately on the symmetric and antisymmetric Lamb modes of a bi-layered structure.

\section{Theory}

We consider a layered structure composed of two identical, isotropic and homogeneous plates with an adhesive layer in between. We denote $\rho$ the mass density 
of the plate and $C_{l}$, the corresponding longitudinal wave velocity, $C_{t}$, the transverse wave velocity, $h$ the plate thickness, $d$ the adhesive thickness with the lateral dimensions supposed infinite. Here $d$ is assumed to be small and the imperfect interface is modeled using the spring model i.e., the structure is modeled as two plates linked by a normal spring of stiffness $K_{N}$ and a tangential spring of stiffness $K_{T}$ Mezil et al. [8]. We consider the two dimensional motion in the $\mathrm{x}-\mathrm{z}$ plane with the harmonic waves propagating in the $\mathrm{x}$ direction and the thickness is in the $\mathrm{z}$ direction. The scalar $\phi$ and the vector potential $\psi$ of displacement, which are satisfied with wave motion equations in the plate can be written as

$$
\begin{aligned}
& \phi=\left(A_{1} \cosh (p z)+A_{2} \sinh (p z)\right) e^{i(k x-\omega t),} \\
& \psi=\left(B_{1} \cosh (q z)+B_{2} \sinh (q z)\right) e^{i(k x-\omega t),}
\end{aligned}
$$

where $k$ is the wave number, $\omega$ the angular velocity, $p^{2}=k^{2}-k_{l}^{2}, q^{2}=k^{2}-k_{t}^{2}$, $k_{l}=\frac{\omega}{C_{l}}, k_{t}=\frac{\omega}{C_{t}}$. The displacement components in the medium of the plate suppressing the common factor $e^{i(k x-\omega t)}$ is given by Nayfeh et al. [10]:

$$
\begin{aligned}
& u_{x}=i k\left(A_{1} \cosh (p z)+A_{2} \sinh (p z)\right)-q\left(B_{1} \sinh (q z)+B_{2} \cosh (q z)\right), \\
& u_{z}=p\left(A_{1} \sinh (p z)+A_{2} \cosh (p z)\right)+i k\left(B_{1} \cosh (q z)+B_{2} \sinh (q z)\right), \\
\tau_{z z}= & \mu\left[\left(k^{2}+q^{2}\right)\left(A_{1} \cosh (p z)+A_{2} \sinh (p z)\right)+2 i q k\left(B_{1} \sinh (q z)+B_{2} \cosh (q z)\right)\right], \\
\tau_{x z}= & \mu\left[2 i p k\left(A_{1} \sinh (q z)+A_{2} \cosh (q z)\right)-\left(k^{2}+q^{2}\right)\left(B_{1} \cosh (q z)+B_{2} \sinh (q z)\right)\right] .
\end{aligned}
$$

For the Lamb wave propagation in the structure, we write the boundary conditions at each interface. At the interface $z= \pm h$,

$$
\begin{aligned}
\tau_{z z} & =0, \\
\tau_{x z} & =0 .
\end{aligned}
$$

At the interface $z=0$, spring boundary conditions are

$$
\begin{aligned}
\tau_{z z} & =K_{N}\left[u_{z}(z=0+)-u_{z}(z=0-)\right], \\
\tau_{x z} & =K_{T}\left[u_{x}(z=0+)-u_{x}(z=0-)\right] .
\end{aligned}
$$

When equations (1)-(4) are used in the equations (5)-(8), we get a $8 \times 8$ homogeneous system, the determinant of which gives the dispersion equation of the guided waves in the structure. Exploiting the symmetry of the structure, the dispersion equation can be split into two characteristic equations $S(\omega, k)=0$ and $A(\omega, k)=0$ which corresponds to symmetric and antisymmetric modes respectively. The dispersion equations are functions of two variables $\omega, k$. 


\section{Numerical Results and Discussions}

Two elastic plates with identical acoustical properties joined by an adhesive is considered. The contact interface which is the adhesive region is assumed to be very small and is modeled as a spring-type interface that has equivalent stiffness $\left(K_{N}\right)$ in the normal direction and in the tangential direction $\left(K_{T}\right)$. In the model, the interface is able to transfer stress continuously, but allows a jump in displacement components. Lamb wave propagation in the structure is considered for different bonding states of the adhesive. The Lamb wave characteristic equation for any possible state can be obtained by letting $K_{N}, K_{T}$ approach appropriate values. The phase velocity of lamb modes are obtained through the characteristic equation and these phase velocities have the potential to differentiate the quality of bonding at the interface.

In Ismaili et al. [1], the evaluation of the bonding quality is carried out experimentally with the quality of glue degraded by addition of grease. In the theoretical comparisons carried out the law of mixtures was used by them to obtain the stiffness constants for different rates of grease in the adhesive layer. In the present work, we consider $0 \%$ and $75 \%$ grease rates in the adhesive film and use the appropriate values of $K_{N}, K_{T}$ to study the influence of these parameters on the symmetric and anti-symmetric modes separately. The values of the stiffness constants used are given in Table 1.

\begin{tabular}{ccc}
\hline & \multicolumn{2}{c}{ Grease Rate } \\
\hline Stiffness constants & $0 \%$ & $75 \%$ \\
\hline$K_{N}\left(N / m^{3}\right)$ & $1.6 \times 10^{13}$ & $4.3 \times 10^{9}$ \\
$K_{T}\left(N / m^{3}\right)$ & $1.4 \times 10^{13}$ & $5.5 \times 10^{9}$ \\
\hline
\end{tabular}

Table 1: Values of interfacial stiffness constants

Figure 1 displays the symmetric Lamb modes of a bilayer plate with $0 \%$ grease rate, while Figure 2 displays the symmetric Lamb mode for the same plate with $75 \%$ grease rate. When the plate thickness is small there is no discernable change in the two graphs. When $h=1.5$ the number of modes in the given frequency range increases and a shift of modes towards the vertical is also seen with increasing bond strength. This is visibly seen in Figure 3. The situation is also similar in the case of anti-symmetric modes as seen from the plots in Figure 4,5 and 6. The effect of the bonding state on the lowest 
antisymmetric mode is seen more prominently in the plots.

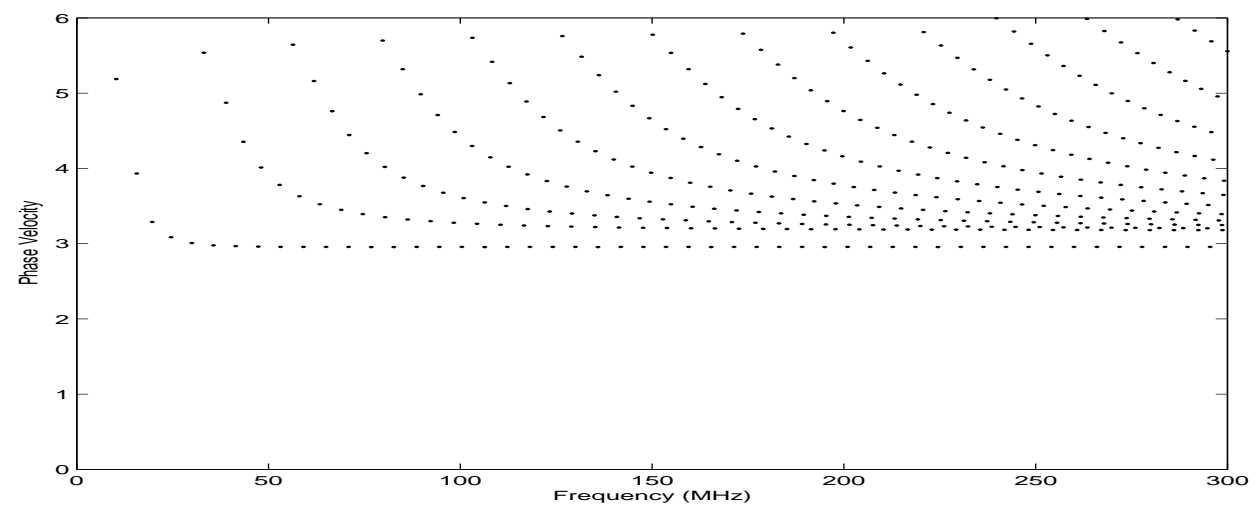

Figure 1: Symmetric Lamb modes with grease rate $0 \%, h=1$ and $K_{n}=1.6 \times 10^{13}$

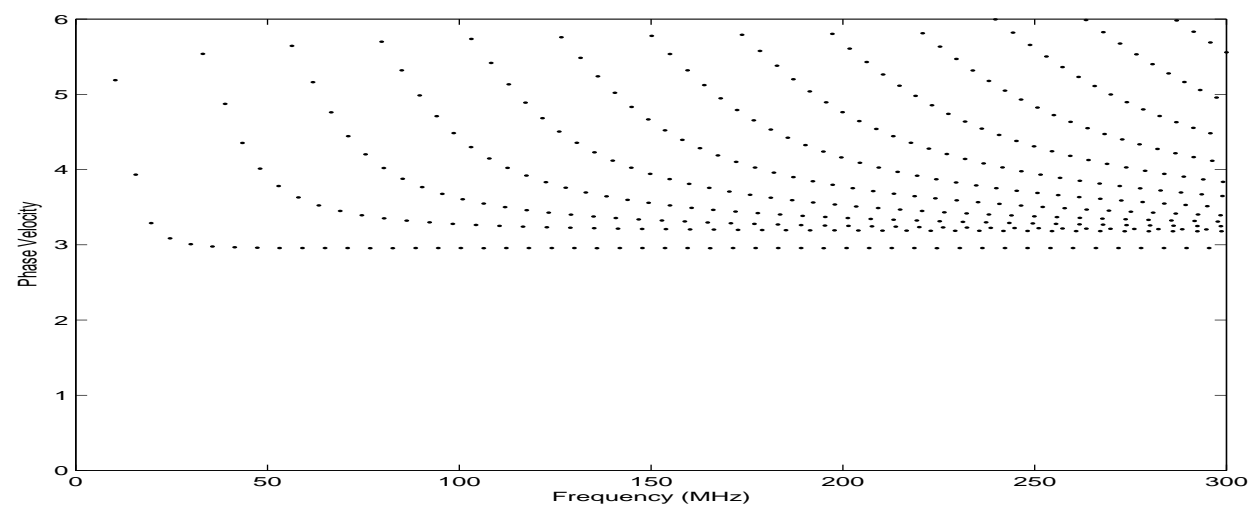

Figure 2: Symmetric Lamb modes with grease rate $75 \%, h=1$ and $K_{n}=4.3 \times 10^{9}$

\section{Conclusion}

The symmetry of the structure helps one to separate the symmetrical modes from the anti-symmetric modes. The influence of the interfacial stiffnesses between the two plates is studied through these modes. The influence of normal stiffness constant on symmetric modes and tangential stiffness constant on antisymmetric modes is visibly seen when the plate thickness is considerable. The 


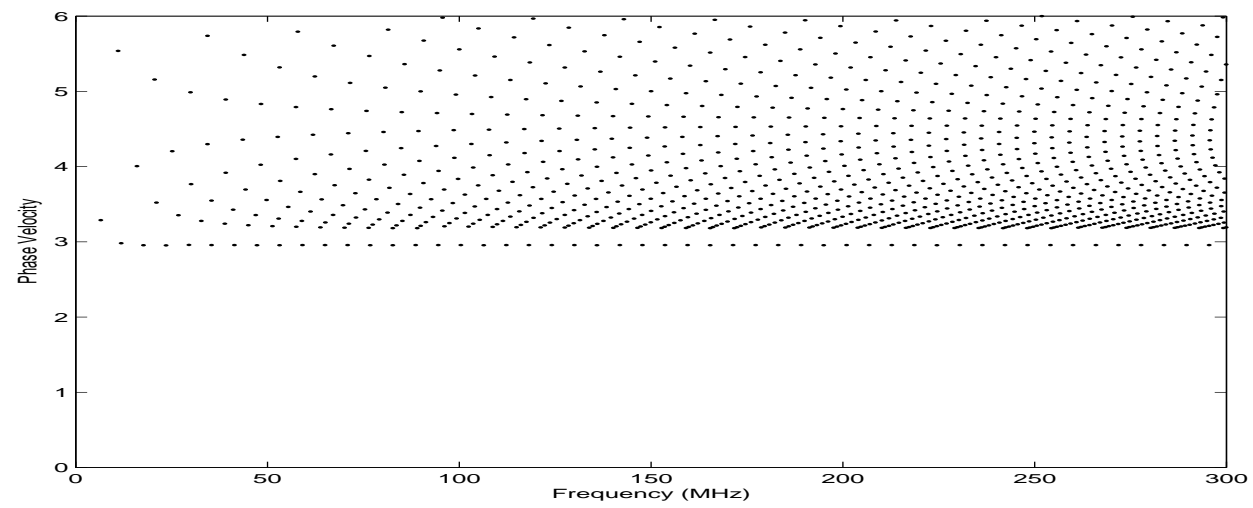

Figure 3: Symmetric Lamb modes with grease rate $75 \%, h=1.5$ and $K_{n}=1.6 \times 10^{13}$

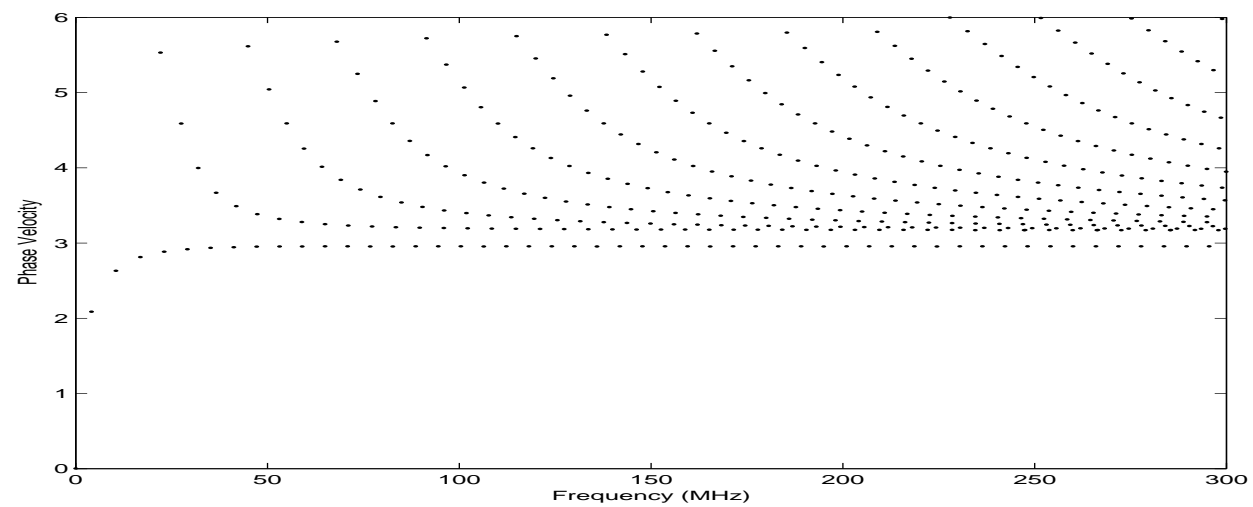

Figure 4: Antisymmetric Lamb modes with grease rate $0 \%, h=1$ and $K_{t}=1.4 \times 10^{13}$ 


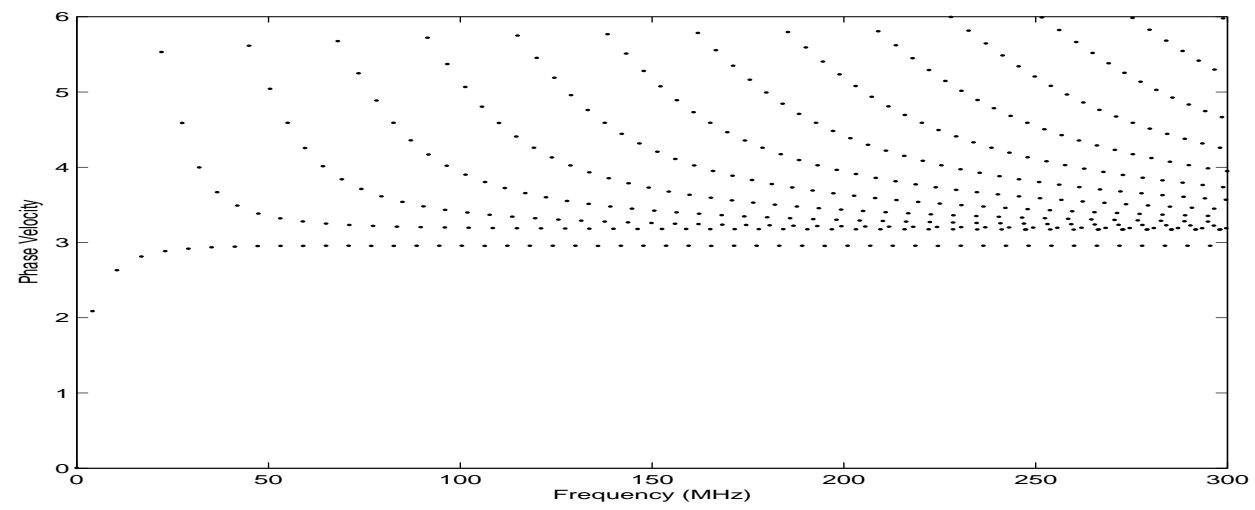

Figure 5: Antisymmetric Lamb modes with grease rate $75 \%, h=1.5$ and $K_{t}=5.5 \times 10^{9}$

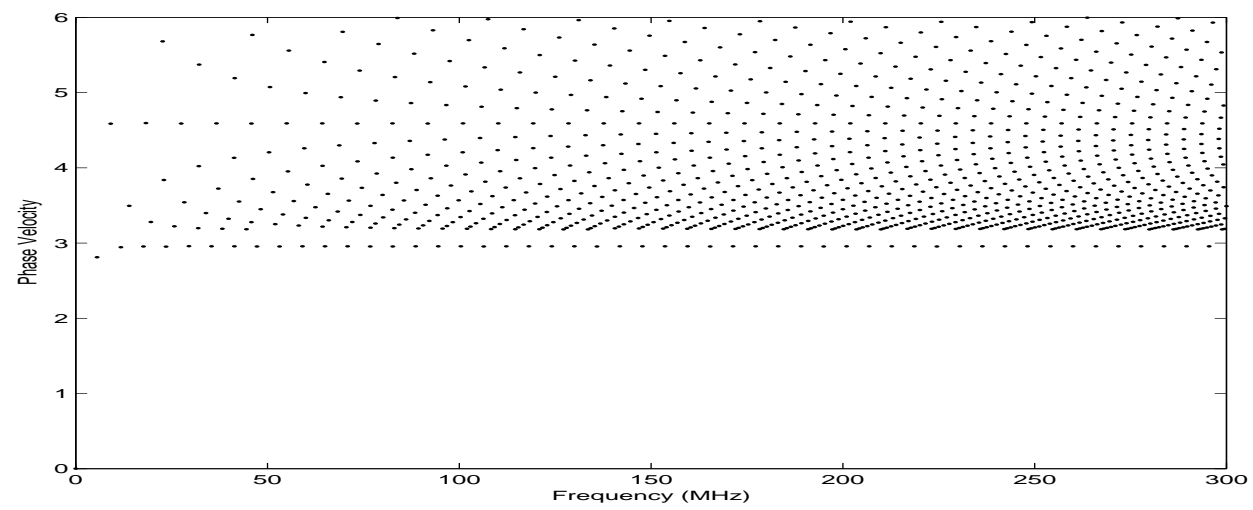

Figure 6: Antisymmetric Lamb modes with $h=1.5$ and $K_{t}=10^{16}$ 
influence of bonding strength on the modes is reflected as an increase in number of modes in a given frequency range or shift of the modes towards the vertical. It is felt that the dispersion spectra would help identify the bonding quality at the interface.

\section{References}

[1] N. Alaoui-Ismaili, C. De Mello-da Silva, M.E. El-Kettani, G. Despaux, M. Roussean and J.L. Izbicki, Lamb modes and acoustic microscopy for the characterization of bonded structures, Proc. Meeting on Acoustics, ICA 2013, June, Moritreel, Canada (2013), 2-7.

[2] J.-M. Baik, R.B. Thompson, Ultrasoinic scattering from imperfect interfaces: A duasi-static model, J. Nondestructive Evaluation, 4, No 3/4 (1984), 177-196.

[3] S. Biwa, A. Suzuki, N. Ohno, Evaluation of interface wave velocity, reflection coefficients and interfacial stiffnesses of contacting surfaces, Ultrasonics, 43 (2005), 495-502.

[4] P.P. Delsanto and M. Scalerandi, A spring model for the simulation of the propagation of ultrasonic pulses through imperfect contact interfaces, $J$. Acoust. Soc. Amer., 104, No 5 (1998), 2584-2591.

[5] Du Jun, C. Jian-chun, Modeling of Laser-Generated Guided waves in bonded plates with a weak interface by the two-layer normal mode expansion method, Chin. Phys. Lett., 20, No 1 (2003), 76-79.

[6] H. Lekesiz, N. Katsube, S.I. Rokhlin, R.R. Seghi, Effective spring stiffness for a periodic array of interacting coplanar penny-shaped cracks at an interface between two dissimilar isotropic materials, Int. J. Solids and Structures, 50 (2013), 2817-2828.

[7] P.A. Martin, Boundary integral equations for the scattering of elastic waves by elastic inclusions with thin interface layers, J. Nondestructive Evaluation, 11, No 3/4 (1992), 167-174.

[8] S. Mezil, J. Laurent, D. Royer, and C. Prada, Non contact probing of interfacial stiffnesses between two plates by zero-group velocity Lamb modes, Applied Physics Letters, 105 (2014), 021605. 
[9] T. Nam, T.Lee, C. Kim, K.-Y. Jhang, N. Kim, Harmonic generation of an obliquely incident ultrasonic wave in solid-solid contact interfaces, Ultrasonics, 52, No 6 (2012), 778-783.

[10] A.H. Nayfeh and P.B. Nagy, Excess attenuation of leaky Lamb waves due to viscous fluid loading, J. Acoust. Soc. Amer., 101, No 5 (1997), 26492658.

[11] Shiuh-Chuan Her and Yi-Chuan Lin, Assessment of adhesive bond strength using the ultrasonic technique, The Journal of Adhesion, 90 (2014), 545554 .

[12] T. Valier-Brasier, T. Dehoux, B. Audoin, Scaled behavior of interface waves at an imperfect solid-solid interface, J. Applied Physics, 112 (2012), 024904 . 
